\title{
Euler and De Moivre's Formulas for Fundamental Matrices of Commutative Quaternions
}

\author{
Hidayet Hüda Kösal* and Tuçe Bilgili \\ (Communicated by Bülent Altunkaya)
}

\begin{abstract}
In this study, Euler and De Moivre's formulas for fundamental matrices of commutative quaternions are obtained. Simple and effective methods are provided to find the powers and roots of these matrices with the aid of De Moivre's formula obtained from the fundamental matrices of commutative quaternions. Moreover, our results are supported by pseudo-codes and some examples.
\end{abstract}

Keywords: Commutative quaternions; fundamental matrices; Euler and De Moivre's formulas.

AMS Subject Classification (2020): 11R52 ; 15A24; $17 A 35$.

\section{Introduction}

In 1892, Segre introduced the concept of commutative quaternions [1]. This number system is sometimes referred to as reduced bi-quaternions [2]. The set of commutative quaternions is a commutative ring under a combination law and commutative law of a four-dimensional Clifford algebra. Also, this set contains non-trivial idempotents, zero-divisors and nilpotent elements [3]. The commutative quaternions play an important role in neural networks, control and system theory, digital signal and image processing, etc. Thus, there is a considerable of literature on commutative quaternions and their matrices in recent years. Pei et al. presented digital image processing based on commutative quaternions [2]. Also, the authors defined a simplified commutative quaternion polar form to represent the color image. In [4], Pei et al. first introduced the eigenvalues, eigenvectors, singular value decomposition and generalized inverse of a commutative quaternion matrix. In [5], Isokawa et al. presented two types of multistate Hopfield neural networks using commutative quaternion. In [6], Kosal et al. developed some explicit expression of the solution of the Kalman-Yakubovichconjugate commutative quaternions matrix equations. In [7], Yuan et al. studied the Hermitian solutions of commutative quaternion matrix equation $(A X B, C X D)=(E, G)$. In [8], Kosal and Tosun constructed universal similarity factorization equalities over the commutative quaternions and their matrices. Also, the authors studied some algebraic properties of commutative quaternions and commutative quaternion matrices. In [9], Kosal derived the expressions of minimal norm least-squares solution for the commutative quaternion matrix equation $A X=B$. Moreover, the author investigated their applications in colour image restoration. In [10], Zhang et al. introduced concepts of norms of commutative quaternion matrices and derived two algebraic techniques for finding solutions of least squares for the matrix equations $A X \approx B$ and $A X C \approx B$ in commutative quaternion matrix algebra.

In this study, Euler and De Moivre's formulas for fundamental matrices of commutative quaternions are obtained. A simple and effective methods is provided to find the powers and roots of these matrices with the aid of De Moivre's formula obtained from the fundamental matrices of commutative quaternions. 
Throughout this paper, the following notations will be used. Let $\mathbb{N}, \mathbb{Z}, \mathbb{R}$ and $\mathbb{H}$ denote the set of natural number, integer numbers, real numbers and commutative quaternions, respectively. Also, all computations are performed on an Intel i7-3630QM@2.40GHz/16 GB computer using MATLAB R2016a software.

\section{Algebraic Properties of Commutative Quaternions}

The set of commutative quaternions can be represented as

$$
\mathbb{H}=\left\{a=a_{0}+a_{1} i+a_{2} j+a_{3} k: a_{0}, a_{1}, a_{2}, a_{3} \in \mathbb{R} \text { and } i, j, k \notin \mathbb{R}\right\}
$$

where

$$
i^{2}=-1, j^{2}=1, k^{2}=-1, i j=j i=k, j k=k j=i, k i=i k=-j .
$$

From (2.2), the operation of multiplication on the set of commutative quaternions $\mathbb{H}$ is commutative. There are three types of conjugates of the $a=a_{0}+a_{1} i+a_{2} j+a_{3} k \in \mathbb{H}$. They are ${ }^{1} \bar{a}=a_{0}-a_{1} i+a_{2} j-a_{3} k,{ }^{2} \bar{a}=$ $a_{0}+a_{1} i-a_{2} j-a_{3} k$ and ${ }^{3} \bar{a}=a_{0}-a_{1} i-a_{2} j+a_{3} k$. The norm of the $a \in \mathbb{H}$ is defined as

$$
\|a\|=\sqrt[4]{a\left({ }^{1} \bar{a}\right)\left({ }^{2} \bar{a}\right)(3 \bar{a})}=\sqrt[4]{\left[\left(a_{0}+a_{2}\right)^{2}+\left(a_{1}+a_{3}\right)^{2}\right]\left[\left(a_{0}-a_{2}\right)^{2}+\left(a_{1}-a_{3}\right)^{2}\right]} .
$$

If $\|a\| \neq 0$ for $a \in \mathbb{H}$ then $a$ has multiplicative inverses. Inverse of $a$ is defined by

$$
a^{-1}=\frac{\left({ }^{1} \bar{a}\right)\left({ }^{2} \bar{a}\right)\left({ }^{3} \bar{a}\right)}{\|a\|^{4}} .
$$

Also, every commutative quaternion $a \in \mathbb{H}(\|a\| \neq 0)$ can be written by

$$
a=\|a\|(\cos \phi+i \sin \phi)(\cosh \theta+j \sinh \theta)(\cos \psi+k \sin \psi)
$$

where $\phi$ and $\psi$ are Euclidean angle, $\theta$ is the hyperbolic angle. Also $\phi, \psi$ and $\theta$ are called principal arguments. Principal arguments for $a \in \mathbb{H}$ are equal to

$$
\begin{aligned}
& \phi=\frac{1}{2} \tan ^{-1}\left(\frac{2\left(a_{0} a_{1}-a_{2} a_{3}\right)}{a_{0}^{2}-a_{1}^{2}-a_{2}^{2}+a_{3}^{2}}\right), \\
& \theta=\frac{1}{2} \tanh ^{-1}\left(\frac{2\left(a_{0} a_{2}+a_{1} a_{3}\right)}{a_{0}^{2}+a_{1}^{2}+a_{2}^{2}+a_{3}^{2}}\right), \\
& \psi=\frac{1}{2} \tan ^{-1}\left(\frac{2\left(a_{0} a_{3}-a_{1} a_{2}\right)}{a_{0}^{2}+a_{1}^{2}-a_{2}^{2}-a_{3}^{2}}\right) .
\end{aligned}
$$

The equality in (2.4) is called the polar representation of the $a \in \mathbb{H}$.

Since $i^{2}=k^{2}=-1$ and $j^{2}=1$ for any $a \in \mathbb{H}(\|a\| \neq 0)$, also we can express generalization of Euler's formula for commutative quaternions as follows

$$
\begin{aligned}
e^{\phi i+\theta j+\psi k}= & {\left[1-\frac{\phi^{2}}{2 !}+\frac{\phi^{4}}{4 !}-\ldots+i\left(\phi-\frac{\phi^{3}}{3 !}+\frac{\phi^{5}}{5 !}-\ldots\right)\right]\left[1+\frac{\theta^{2}}{2 !}+\frac{\theta^{4}}{4 !}+\ldots+j\left(\theta+\frac{\theta^{3}}{3 !}+\frac{\theta^{5}}{5 !}+\ldots\right)\right] } \\
& {\left[1-\frac{\psi^{2}}{2 !}+\frac{\psi^{4}}{4 !}-\ldots+k\left(\psi-\frac{\psi^{3}}{3 !}+\frac{\psi^{5}}{5 !}-\ldots\right)\right] } \\
= & (\cos \phi+i \sin \phi)(\cosh \theta+j \sinh \theta)(\cos \psi+k \sin \psi)
\end{aligned}
$$

for any real $\phi, \theta$ and $\psi$. Thus any $a \in \mathbb{H}(\|a\| \neq 0)$ can be written by

$$
a=\|a\| e^{\phi i+\theta j+\psi k} .
$$

The equality in (2.6) is called the Euler form of the commutative quaternion $a \in \mathbb{H}[11]$.

Theorem 2.1. [11] Let $a=\|a\|(\cos \phi+i \sin \phi)(\cosh \theta+j \sinh \theta)(\cos \psi+k \sin \psi)=\|a\| e^{\phi i+\theta j+\psi k}$. Then we have

$$
a^{n}=\|a\|^{n}(\cos n \phi+i \sin n \phi)(\cosh n \theta+j \sinh n \theta)(\cos n \psi+k \sin n \psi)=\|a\|^{n} e^{n \phi i+n \theta j+n \psi k}
$$

for every integer $n$. 
Theorem 2.2. [4] Let $a \in \mathbb{H}$. Then the equation $x^{n}=a$ has $n^{2} n^{\text {th }}$ roots for $\forall n \in \mathbb{N}$.

Theorem 2.3. [8] Let $a \in \mathbb{H}$. Then a satisfy the following Universal Similarity Factorization Equality (USFE)

$$
\operatorname{Pdiag}\left(a,{ }^{1} \bar{a},{ }^{2} \bar{a},{ }^{3} \bar{a}\right) P^{-1}=\left(\begin{array}{rrrr}
a_{0} & -a_{1} & a_{2} & -a_{3} \\
a_{1} & a_{0} & a_{3} & a_{2} \\
a_{2} & -a_{3} & a_{0} & -a_{1} \\
a_{3} & a_{2} & a_{1} & a_{0}
\end{array}\right)
$$

where

$$
P=\frac{1}{2}\left(\begin{array}{rrrr}
1 & 1 & 1 & 1 \\
-i & i & -i & i \\
j & j & -j & -j \\
-k & k & k & -k
\end{array}\right) \text { and } P^{-1}=\frac{1}{2}\left(\begin{array}{rrrr}
1 & i & j & k \\
1 & -i & j & -k \\
1 & i & -j & -k \\
1 & -i & -j & k
\end{array}\right)
$$

In here, $\varphi(a)=\left(\begin{array}{rrrr}a_{0} & -a_{1} & a_{2} & -a_{3} \\ a_{1} & a_{0} & a_{3} & a_{2} \\ a_{2} & -a_{3} & a_{0} & -a_{1} \\ a_{3} & a_{2} & a_{1} & a_{0}\end{array}\right)$ is called fundamental matrix of $a \in \mathbb{H}$. USFE over the commutative quaternions clearly reveals three basic facts:

1. $a \in \mathbb{H}$ is algebraically isomorphic to

$$
\mathbb{H}^{\prime}=\left\{\left(\begin{array}{rrrr}
a_{0} & -a_{1} & a_{2} & -a_{3} \\
a_{1} & a_{0} & a_{3} & a_{2} \\
a_{2} & -a_{3} & a_{0} & -a_{1} \\
a_{3} & a_{2} & a_{1} & a_{0}
\end{array}\right): a_{0}, a_{1}, a_{2}, a_{3} \in \mathbb{R}\right\} \subset \mathbb{R}^{4 \times 4}
$$

through the bijective map $a \in \mathbb{H}$ defined as

$$
a=a_{0}+a_{1} i+a_{2} j+a_{3} k \rightarrow \varphi(a)=\left(\begin{array}{rrrr}
a_{0} & -a_{1} & a_{2} & -a_{3} \\
a_{1} & a_{0} & a_{3} & a_{2} \\
a_{2} & -a_{3} & a_{0} & -a_{1} \\
a_{3} & a_{2} & a_{1} & a_{0}
\end{array}\right)
$$

2. Every $a \in \mathbb{H}$ has a real matrix representation

$$
\varphi(a)=\left(\begin{array}{rrrr}
a_{0} & -a_{1} & a_{2} & -a_{3} \\
a_{1} & a_{0} & a_{3} & a_{2} \\
a_{2} & -a_{3} & a_{0} & -a_{1} \\
a_{3} & a_{2} & a_{1} & a_{0}
\end{array}\right)
$$

over the $\mathbb{R}$.

3. All real matrices in $\mathbb{H}^{\prime}$ can uniformly be diagonalized over the commutative quaternions.

Theorem 2.4. [11] Let $a, b \in \mathbb{H}$ and $\lambda \in \mathbb{R}$. Then the following identities are satisfied:

1. $\varphi(a+b)=\varphi(a)+\varphi(b)$,

2. $\varphi(a b)=\varphi(a) \varphi(b)$,

3. $\varphi(\varphi(a) b)=\varphi(a) \varphi(b)$,

4. $\varphi(\lambda a)=\lambda \varphi(a)$,

5. $(\varphi(a))^{T}=\varphi\left({ }^{1} \bar{a}\right)$,

6. $\operatorname{trace}(\varphi(a))=a+{ }^{1} \bar{a}+{ }^{2} \bar{a}+{ }^{3} \bar{a}$,

7. $\|a\|^{4}=|\operatorname{det}(\varphi(a))|$. 


\section{Euler's and De Moivre's Formulas for the Fundamental Matrices of Commutative Quaternions}

Now, we introduce the Euler and De Moivre's formulas for fundamental matrix $\varphi(a)$ of $a \in \mathbb{H}(\|a\| \neq 0)$. Depending on the casual character of commutative quaternions, we can express fundamental matrix $\varphi(a)$ as follows:

$$
\varphi(a)=\sqrt[4]{(\operatorname{det}(\varphi(a)))}\left(\cos \phi I_{4}+\varphi(i) \sin \phi\right)\left(\cosh \theta I_{4}+\varphi(j) \sinh \theta\right)\left(\cos \psi I_{4}+\varphi(k) \sin \psi\right)
$$

where $I_{4}$ is the $4 \times 4$ unit matrix,

$$
\begin{gathered}
\cos \phi I_{4}+\varphi(i) \sin \phi=\left(\begin{array}{rrrr}
\cos \phi & -\sin \phi & 0 & 0 \\
\sin \phi & \cos \phi & 0 & 0 \\
0 & 0 & \cos \phi & -\sin \phi \\
0 & 0 & \sin \phi & \cos \phi
\end{array}\right), \\
\cosh \theta I_{4}+\varphi(j) \sinh \theta=\left(\begin{array}{rrrr}
\cosh \theta & 0 & \sinh \theta & 0 \\
0 & \cosh \theta & 0 & \sinh \theta \\
\sinh \theta & 0 & \cosh \theta & 0 \\
0 & \sinh \theta & 0 & \cosh \theta
\end{array}\right)
\end{gathered}
$$

and

$$
\cos \psi I_{4}+\varphi(k) \sin \psi=\left(\begin{array}{rrrr}
\cos \psi & 0 & 0 & -\sin \psi \\
0 & \cos \psi & \sin \psi & 0 \\
0 & -\sin \psi & \cos \psi & 0 \\
\sin \psi & 0 & 0 & \cos \psi
\end{array}\right)
$$

The equality in the (3.1) is called the polar form of the fundamental matrix $\varphi(a)$.

Theorem 3.1. Let $\phi, \theta, \psi \in \mathbb{R}$ and $n \in \mathbb{Z}$. Then following identities are satisfied:

a. $\left(\cos \phi I_{4}+\varphi(i) \sin \phi\right)^{n}=\cos n \phi I_{4}+\varphi(i) \sin n \phi$,

b. $\left(\cosh \theta I_{4}+\varphi(j) \sinh \theta\right)^{n}=\cosh n \theta I_{4}+\varphi(j) \sinh n \theta$,

c. $\left(\cos \psi I_{4}+\varphi(k) \sin \psi\right)^{n}=\cos n \psi I_{4}+\varphi(k) \sin n \psi$.

Proof. We use induction on positive integer $n$. Suppose that

$$
\left(\cos \phi I_{4}+\varphi(i) \sin \phi\right)^{k}=\left(\begin{array}{cccc}
\cos k \phi & -\sin k \phi & 0 & 0 \\
\sin k \phi & \cos k \phi & 0 & 0 \\
0 & 0 & \cos k \phi & -\sin k \phi \\
0 & 0 & \sin k \phi & \cos k \phi
\end{array}\right)
$$

Using the identities

$$
\begin{aligned}
& \cos \phi \cos n \phi-\sin \phi \sin n \phi=\cos (n+1) \phi \\
& \cos \phi \sin n \phi+\sin \phi \cos n \phi=\sin (n+1) \phi
\end{aligned}
$$

we get 


$$
\begin{aligned}
\left(\cos \phi I_{4}\right. & +\varphi(i) \sin \phi)^{k+1}=\left(\cos \phi I_{4}+\varphi(i) \sin \phi\right)^{k}\left(\cos \phi I_{4}+\varphi(i) \sin \phi\right) \\
& =\left(\begin{array}{cccc}
\cos k \phi & -\sin k \phi & 0 & 0 \\
\sin k \phi & \cos k \phi & 0 & 0 \\
0 & 0 & \cos k \phi & -\sin k \phi \\
0 & 0 & \sin k \phi & \cos k \phi
\end{array}\right)\left(\begin{array}{cccc}
\cos \phi & -\sin \phi & 0 & 0 \\
\sin \phi & \cos \phi & 0 & 0 \\
0 & 0 & \cos \phi & -\sin \phi \\
0 & 0 & \sin \phi & \cos \phi
\end{array}\right) \\
& =\left(\begin{array}{cccc}
\cos (k+1) \phi & -\sin (k+1) \phi & 0 & 0 \\
\sin (k+1) \phi & \cos (k+1) \phi & 0 & 0 \\
0 & 0 & \cos (k+1) \phi & -\sin (k+1) \phi \\
0 & 0 & \sin (k+1) \phi & \cos (k+1) \phi
\end{array}\right) \\
& =\cos (k+1) \phi I_{4}+\varphi(i) \sin (k+1) \phi .
\end{aligned}
$$

Hence the first formula is true for $\forall n \in \mathbb{N}$. Also the formula hold for all integer $n$, since

$$
\varphi(a)^{-1}=\frac{1}{\sqrt[4]{(\operatorname{det}(\varphi(a)))}}\left(\cos \phi I_{4}-\varphi(i) \sin \phi\right)\left(\cosh \theta I_{4}-\varphi(j) \sinh \theta\right)\left(\cos \psi I_{4}-\varphi(k) \sin \psi\right) .
$$

The accuracy of $b$ and $c$ are also shown in a similar way.

Corollary 3.1. (De Moivre's Formula): Let $\varphi(a)$ be fundamental matrix of $a \in \mathbb{H}(\|a\| \neq 0)$. Then, we have

$$
\varphi(a)^{n}=(\sqrt[4]{(\operatorname{det}(\varphi(a)))})^{n}\left(\cos n \phi I_{4}+\varphi(i) \sin n \phi\right)\left(\cosh n \theta I_{4}+\varphi(j) \sinh n \theta\right)\left(\cos n \psi I_{4}+\varphi(k) \sin n \psi\right)
$$

for every $n \in \mathbb{Z}$.

Theorem 3.2. Let $\varphi(a)$ be fundamental matrix of commutative quaternion $a=a_{0}+a_{1} i+a_{2} j+a_{3} k$. The equation $X^{n}=\varphi(a)$ has $n^{2} n^{\text {th }}$ roots for $\forall n \in \mathbb{N}$ and these roots are in this form

$$
\begin{aligned}
X=(\varphi(a))^{\frac{1}{n}} & =\left(\operatorname{det}\left(\varphi\left(a_{0}+a_{2}+\left(a_{1}+a_{3}\right) i\right)\right)\right)^{\frac{1}{4 n}} \varphi\left(\cos \frac{\phi+2 \pi k_{1}}{n}+i \sin \frac{\phi+2 \pi k_{1}}{n}\right)^{\frac{1}{n}} \varphi\left(\frac{1+j}{2}\right) \\
& +\left(\operatorname{det}\left(\varphi\left(a_{0}-a_{2}+\left(a_{1}-a_{3}\right) i\right)\right)\right)^{\frac{1}{4 n}} \varphi\left(\cos \frac{\phi^{\prime}+2 \pi k_{2}}{n}+i \sin \frac{\phi^{\prime}+2 \pi k_{2}}{n}\right)^{\frac{1}{n}} \varphi\left(\frac{1-j}{2}\right) .
\end{aligned}
$$

where $k_{1}, k_{2}=0,1, \ldots, n-1$ and $\phi, \phi^{\prime}$ are arguments of complex numbers $a_{0}+a_{2}+\left(a_{1}+a_{3}\right) i$ and $a_{0}-a_{2}+$ $\left(a_{1}-a_{3}\right) i$, respectively.

Proof. We can express the commutative quaternion $a=a_{0}+a_{1} i+a_{2} j+a_{3} k$ as follows

$$
\begin{aligned}
a & =\left(a_{0}+a_{1} i\right)+\left(a_{2}+a_{3} i\right) j \\
& =\left(a_{0}+a_{2}+\left(a_{1}+a_{3}\right) i\right) \frac{1+j}{2}+\left(a_{0}-a_{2}+\left(a_{1}-a_{3}\right) i\right) \frac{1-j}{2} .
\end{aligned}
$$

In this case, we have

$$
\begin{aligned}
\varphi(a) & =\varphi\left(a_{0}+a_{2}+\left(a_{1}+a_{3}\right) i\right) \varphi\left(\frac{1+j}{2}\right)+\varphi\left(a_{0}-a_{2}+\left(a_{1}-a_{3}\right) i\right) \varphi\left(\frac{1-j}{2}\right) \\
& =\left(\operatorname{det}\left(\varphi\left(a_{0}+a_{2}+\left(a_{1}+a_{3}\right) i\right)\right)\right)^{\frac{1}{4}} \varphi(\cos \phi+i \sin \phi) \varphi\left(\frac{1+j}{2}\right) \\
& +\left(\operatorname{det}\left(\varphi\left(a_{0}-a_{2}+\left(a_{1}-a_{3}\right) i\right)\right)\right)^{\frac{1}{4}} \varphi\left(\cos \phi^{\prime}+i \sin \phi^{\prime}\right) \varphi\left(\frac{1-j}{2}\right) .
\end{aligned}
$$

In here, $\varphi\left(\frac{1+j}{2}\right)$ and $\varphi\left(\frac{1-j}{2}\right)$ matrices are idempotent and $\forall n \in \mathbb{N}$

$$
\left(\varphi\left(\frac{1+j}{2}\right)\right)^{n}=\left(\varphi\left(\frac{1+j}{2}\right)\right)^{\frac{1}{n}}=\varphi\left(\frac{1+j}{2}\right),\left(\varphi\left(\frac{1-j}{2}\right)\right)^{n}=\left(\varphi\left(\frac{1-j}{2}\right)\right)^{\frac{1}{n}}=\varphi\left(\frac{1-j}{2}\right) .
$$

In this case, we get

$$
\begin{aligned}
(\varphi(a))^{\frac{1}{n}} & =\left(\operatorname{det}\left(\varphi\left(a_{0}+a_{2}+\left(a_{1}+a_{3}\right) i\right)\right)\right)^{\frac{1}{4 n}} \varphi(\cos \phi+i \sin \phi)^{\frac{1}{n}} \varphi\left(\frac{1+j}{2}\right)^{\frac{1}{n}} \\
& +\left(\operatorname{det}\left(\varphi\left(a_{0}-a_{2}+\left(a_{1}-a_{3}\right) i\right)\right)\right)^{\frac{1}{4 n}} \varphi\left(\cos \phi^{\prime}+i \sin \phi^{\prime}\right)^{\frac{1}{n}} \varphi\left(\frac{1-j}{2}\right)^{\frac{1}{n}} \\
& =\left(\operatorname{det}\left(\varphi\left(a_{0}+a_{2}+\left(a_{1}+a_{3}\right) i\right)\right)\right)^{\frac{1}{4 n}} \varphi(\cos \phi+i \sin \phi)^{\frac{1}{n}} \varphi\left(\frac{1+j}{2}\right) \\
& +\left(\operatorname{det}\left(\varphi\left(a_{0}-a_{2}+\left(a_{1}-a_{3}\right) i\right)\right)\right)^{\frac{1}{4 n}} \varphi\left(\cos \phi^{\prime}+i \sin \phi^{\prime}\right)^{\frac{1}{n}} \varphi\left(\frac{1-j}{2}\right) .
\end{aligned}
$$


Since the complex numbers $(\cos \phi+i \sin \phi)^{\frac{1}{n}}$ and $\left(\cos \phi^{\prime}+i \sin \phi^{\prime}\right)^{\frac{1}{n}}$ will have at most $n$ roots, the equation $X^{n}=\varphi(a)$ has $n^{2} n^{\text {th }}$ roots for $\forall n \in \mathbb{N}$.

\subsection{Euler's Formula for Fundamental Matrix of Commutative Quaternions}

Let $A=\varphi(i) \varphi(j) \varphi(k)$. Obviously, $(\varphi(i))^{2}=-I_{4},(\varphi(j))^{2}=I_{4},(\varphi(k))^{2}=-I_{4}$. Since

$$
\begin{aligned}
e^{\varphi(i) \phi} & =I_{4}+\varphi(i) \phi+\frac{(\varphi(i) \phi)^{2}}{2 !}+\frac{(\varphi(i) \phi)^{3}}{3 !}+\frac{(\varphi(i) \phi)^{4}}{4 !}+\ldots \\
& =I_{4}+\varphi(i) \phi-\frac{\phi^{2}}{2 !} I_{4}-\varphi(i) \frac{\phi^{3}}{3 !}+\frac{\phi^{4}}{4 !} I_{4}+\ldots \\
& =\left(1-\frac{\phi^{2}}{2 !}+\frac{\phi^{4}}{4 !}-\ldots\right) I_{4}+\varphi(i)\left(\phi-\frac{\phi^{3}}{3 !}+\frac{\phi^{5}}{5 !}-\ldots\right) \\
& =\cos \phi I_{4}+\varphi(i) \sin \phi \\
e^{\varphi(j) \theta} & =I_{4}+\varphi(j) \theta+\frac{(\varphi(j) \theta)^{2}}{2 !}+\frac{(\varphi(j) \theta)^{3}}{3 !}+\frac{(\varphi(j) \theta)^{4}}{4 !}+\ldots \\
& =I_{4}+\varphi(j) \theta+\frac{\theta^{2}}{2 !} I_{4}+\varphi(j) \frac{\theta^{3}}{3 !}+\frac{\theta^{4}}{4 !} I_{4}+\ldots \\
& =\left(1+\frac{\theta^{2}}{2 !}+\frac{\theta^{4}}{4 !}+\ldots\right) I_{4}+\varphi(j)\left(\theta+\frac{\theta^{3}}{3 !}+\frac{\theta^{5}}{5 !}+\ldots\right) \\
& =\cosh \theta I_{4}+\varphi(j) \sinh \theta
\end{aligned}
$$

and

$$
\begin{aligned}
e^{\varphi(k) \psi} & =I_{4}+\varphi(k) \psi+\frac{(\varphi(k) \psi)^{2}}{2 !}+\frac{(\varphi(k) \psi)^{3}}{3 !}+\frac{(\varphi(k) \psi)^{4}}{4 !}+\ldots \\
& =I_{4}+\varphi(k) \psi-\frac{\psi^{2}}{2 !} I_{4}-\varphi(k) \frac{\psi^{3}}{3 !}+\frac{\psi^{4}}{4 !} I_{4}+\ldots \\
& =\left(1-\frac{\psi^{2}}{2 !}+\frac{\psi^{4}}{4 !}-\ldots\right) I_{4}+\varphi(k)\left(\psi-\frac{\psi^{3}}{3 !}+\frac{\psi^{5}}{5 !}-\ldots\right) \\
& =\cos \psi I_{4}+\varphi(k) \sin \psi
\end{aligned}
$$

we have

$$
e^{\varphi(i) \phi+\varphi(j) \theta+\varphi(k) \psi}=\left(\cos \phi I_{4}+\varphi(i) \sin \phi\right)\left(\cosh \theta I_{4}+\varphi(j) \sinh \theta\right)\left(\cos \psi I_{4}+\varphi(k) \sin \psi\right)
$$

Thus, every fundamental matrix of commutative quaternion $a(\|a\| \neq 0)$ can be written by

$$
\varphi(a)=\sqrt[4]{(\operatorname{det}(\varphi(a)))} e^{\varphi(i) \phi+\varphi(j) \theta+\varphi(k) \psi} .
$$

This equality is called the Euler formula of the fundamental matrix $\varphi(a)$.

Corollary 3.2. Let $\varphi(a)=\sqrt[4]{(\operatorname{det}(\varphi(a)))} e^{\varphi(i) \phi+\varphi(j) \theta+\varphi(k) \psi}$ and $\varphi(b)=\sqrt[4]{(\operatorname{det}(\varphi(b)))} e^{\varphi(i) \phi^{\prime}+\varphi(j) \theta^{\prime}+\varphi(k) \psi^{\prime}}$ are fundamental matrices of commutative quaternions $a, b(\|a\| \neq 0,\|b\| \neq 0)$. Then we have

$$
\begin{aligned}
& \varphi(a)^{n}=\sqrt[4]{(\operatorname{det}(\varphi(a)))^{n}} e^{n \varphi(i) \phi+n \varphi(j) \theta+n \varphi(k) \psi}, \quad n \in \mathbb{Z}, \\
& \varphi(a)^{-1}=\sqrt[4]{(\operatorname{det}(\varphi(a)))^{-1}} e^{-\varphi(i) \phi-\varphi(j) \theta-\varphi(k) \psi}
\end{aligned}
$$

and

$$
\varphi(a) \varphi(b)=\sqrt[4]{\operatorname{det}(\varphi(a b))} e^{\varphi(i)\left(\phi+\phi^{\prime}\right)+\varphi(j)\left(\theta+\theta^{\prime}\right)+\varphi(k)\left(\psi+\psi^{\prime}\right)} .
$$




\subsection{Numerical algorithms for Power and Root Calculation of Fundamental Matrices}

We now give the numerical algorithms for obtaining the power and root of fundamental matrices based on our results.

\section{Algorithm 1.}

1. Begin

2. Enter the fundamental matrix and the power to calculate the fundamental matrix

3. Calculate the determinant of the fundamental matrix

4. Calculate fi, teta, psi angles according to equation (2.5)

5. Calculate Euler's formula according to equation (3.4)

6. Calculate the power of the fundamental matrix according to Corollary 3.2.

7. Write power

8. Stop.

\section{Algorithm 2.}

1. Begin

2. Enter the fundamental matrix and the root of the basic matrix to be calculated

3. Rewrite the fundamental matrix according to the equation (3.2)

4. Calculate the roots of the fundamental matrix according to Theorem 3.2

5. Write Roots

6. Stop.

The graph below shows the time elapsed when calculating the power of a randomly generated fundamental matrix with the matrix multiplication and the algorithm 1 in MATLAB. 


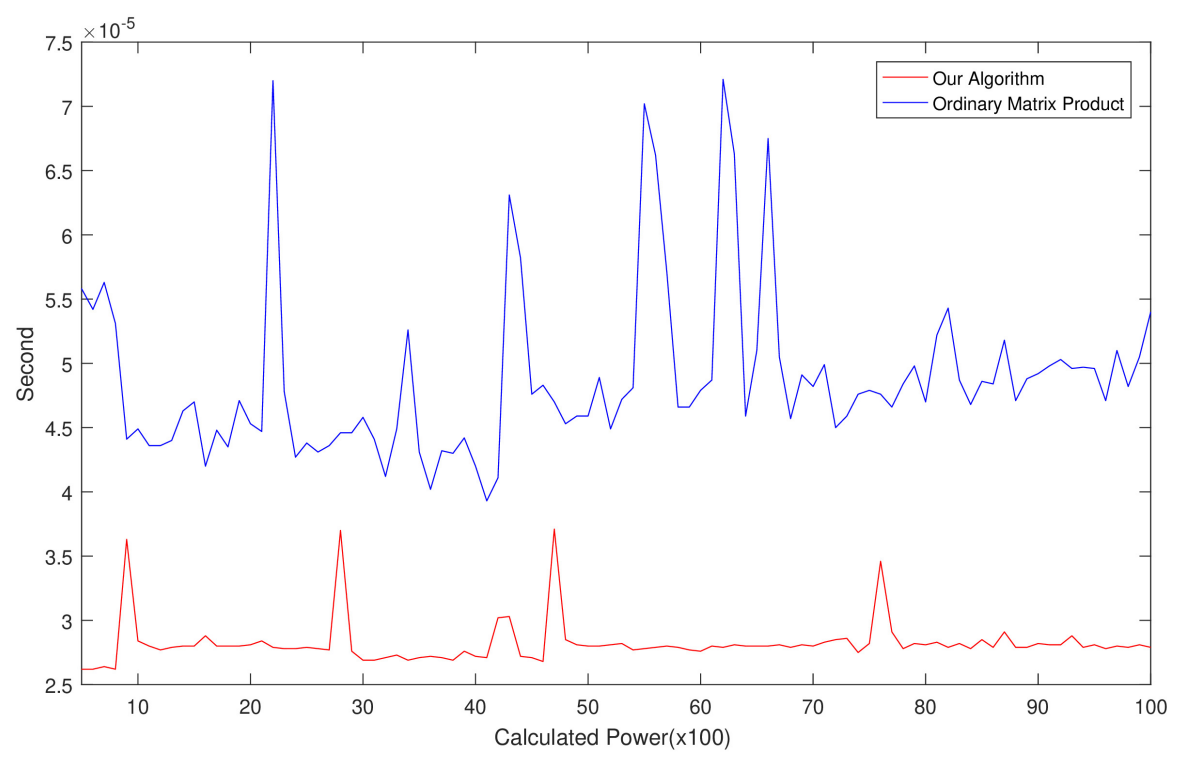

Figure 1. Comparison of ordinary matrix product and algorithm 1 methods.

It is clear from the graph that the algorithm 1 used to calculate the power of the fundamental matrix reaches the result in a shorter time than the ordinary matrix multiplication.

\subsection{Numerical Examples}

Example 3.1. Let us find the 100th power of the fundamental matrix

$$
A=\left(\begin{array}{rrrr}
\frac{1}{2} & -\frac{1}{2} & \frac{1}{2} & \frac{1}{2} \\
\frac{1}{2} & \frac{1}{2} & -\frac{1}{2} & \frac{1}{2} \\
\frac{1}{2} & \frac{1}{2} & \frac{1}{2} & -\frac{1}{2} \\
-\frac{1}{2} & \frac{1}{2} & \frac{1}{2} & \frac{1}{2}
\end{array}\right)
$$

according to the Corollary 3.2.

The determinant of matrix $A$ is 1 . Let us now obtain the Euler formula of the matrix $A$. Since

$$
\begin{aligned}
& \phi=\frac{1}{2} \tan ^{-1}\left(\frac{2\left(a_{0} a_{1}-a_{2} a_{3}\right)}{a_{0}^{2}-a_{1}^{2}-a_{2}^{2}+a_{3}^{2}}\right)=\frac{1}{2} \tan ^{-1}\left(\frac{1}{0}\right)=\frac{\pi}{4}, \\
& \theta=\frac{1}{2} \tanh ^{-1}\left(\frac{2\left(a_{0} a_{2}+a_{1} a_{3}\right)}{a_{0}^{2}+a_{1}^{2}+a_{2}^{2}+a_{3}^{2}}\right)=\frac{1}{2} \tanh ^{-1}(0)=0
\end{aligned}
$$

and

$$
\psi=\frac{1}{2} \tan ^{-1}\left(\frac{2\left(a_{0} a_{3}-a_{1} a_{2}\right)}{a_{0}^{2}+a_{1}^{2}-a_{2}^{2}-a_{3}^{2}}\right)=\frac{1}{2} \tan ^{-1}\left(\frac{-1}{0}\right)=\frac{\pi}{4}
$$

the Euler formula of matrix $A$ is

$$
A=\sqrt[4]{\operatorname{det}(A)} e^{\varphi(i) \phi+\varphi(j) \theta+\varphi(k) \psi}=e^{\varphi(i) \frac{\pi}{4}+\varphi(k) \frac{\pi}{4}} .
$$

Therefore we get

$$
A^{100}=e^{100 \varphi(i) \frac{\pi}{4}+100 \varphi(k) \frac{\pi}{4}}=e^{100 \varphi(i) \frac{\pi}{4}} e^{100 \varphi(k) \frac{\pi}{4}}=\left(-I_{4}\right)\left(-I_{4}\right)=I_{4}
$$

according to Corollary 3.2 where $I_{4}$ is the $4 \times 4$ unit.

Example 3.2. Let us find the square root of the fundamental matrix

$$
A=\left(\begin{array}{rrrr}
\frac{1}{2} & -\frac{1}{2} & \frac{1}{2} & \frac{1}{2} \\
\frac{1}{2} & \frac{1}{2} & -\frac{1}{2} & \frac{1}{2} \\
\frac{1}{2} & \frac{1}{2} & \frac{1}{2} & -\frac{1}{2} \\
-\frac{1}{2} & \frac{1}{2} & \frac{1}{2} & \frac{1}{2}
\end{array}\right)
$$


according to the Theorem 3.2.

We can express matrix $A$ as follows:

$$
A=\left(\begin{array}{llll}
1 & 0 & 0 & 0 \\
0 & 1 & 0 & 0 \\
0 & 0 & 1 & 0 \\
0 & 0 & 0 & 1
\end{array}\right) \varphi\left(\frac{1+j}{2}\right)+\left(\begin{array}{rrrr}
0 & -1 & 0 & 0 \\
1 & 0 & 0 & 0 \\
0 & 0 & 0 & -1 \\
0 & 0 & 1 & 0
\end{array}\right) \varphi\left(\frac{1-j}{2}\right)
$$

Then we get

$$
A=\varphi(\cos 0+i \sin 0) \varphi\left(\frac{1+j}{2}\right)+\varphi\left(\cos \frac{\pi}{2}+i \sin \frac{\pi}{2}\right) \varphi\left(\frac{1-j}{2}\right) .
$$

As a result, the following result is obtained

$$
A^{\frac{1}{2}}=\varphi\left(\cos \left(\frac{2 \pi k_{1}}{2}\right)+i \sin \left(\frac{2 \pi k_{1}}{2}\right)\right) \varphi\left(\frac{1+j}{2}\right)+\varphi\left(\cos \left(\frac{\frac{\pi}{2}+2 \pi k_{2}}{2}\right)+i \sin \left(\frac{\frac{\pi}{2}+2 \pi k_{2}}{2}\right)\right) \varphi\left(\frac{1-j}{2}\right)
$$

where $k_{1}, k_{2}=0,1$.

According to Theorem 3.2, the equation $X^{2}=A$ has 4 square roots and these roots are in this form

$$
\begin{gathered}
A^{\frac{1}{2}}= \pm\left(\begin{array}{rrrr}
\frac{2+\sqrt{2}}{4} & -\frac{\sqrt{2}}{4} & \frac{2-\sqrt{2}}{4} & \frac{\sqrt{2}}{4} \\
\frac{\sqrt{2}}{4} & \frac{2+\sqrt{2}}{4} & -\frac{\sqrt{2}}{4} & \frac{2-\sqrt{2}}{4} \\
\frac{2-\sqrt{2}}{4} & \frac{\sqrt{2}}{4} & \frac{2+\sqrt{2}}{4} & -\frac{\sqrt{2}}{4} \\
-\frac{\sqrt{2}}{4} & \frac{2-\sqrt{2}}{4} & \frac{\sqrt{2}}{4} & \frac{2+\sqrt{2}}{4}
\end{array}\right) \\
A^{\frac{1}{2}}= \pm\left(\begin{array}{rrrr}
\frac{2-\sqrt{2}}{4} & \frac{\sqrt{2}}{4} & \frac{2+\sqrt{2}}{4} & -\frac{\sqrt{2}}{4} \\
-\frac{\sqrt{2}}{4} & \frac{2-\sqrt{2}}{4} & \frac{\sqrt{2}}{4} & \frac{2+\sqrt{2}}{4} \\
\frac{2+\sqrt{2}}{4} & -\frac{\sqrt{2}}{4} & \frac{2-\sqrt{2}}{4} & \frac{\sqrt{2}}{4} \\
\frac{\sqrt{2}}{4} & \frac{2+\sqrt{2}}{4} & -\frac{\sqrt{2}}{4} & \frac{2-\sqrt{2}}{4}
\end{array}\right) .
\end{gathered}
$$

\section{Acknowledgments}

The authors would like to thank the anonymous referees for their helpful suggestions and comments which improved significantly the presentation of the paper.

\section{References}

[1] Segre, C.: The real representations of complex elements and extension to bicomplex. Systems. Math. Ann., 40, 413, (1892).

[2] Pei, S. C., Chang, J. H., Ding, J. J.: Commutative reduced biquaternions and their fourier transform for signal and image processing applications. IEEE Trans. on Signal Proces., 52(7), 2012-2031, (2004).

[3] Catoni, F., Boccaletti, D., Cannata, R., Catoni, V., Nichelatti, E., Zampetti, P.: The mathematics of minkowski space-time with an introduction to commutative hypercomplex numbers. Birkhauser Verlag AG, Berlin, (2008).

[4] Pei S., Chang J., Ding J., Chen M.: Eigenvalues and singular value decompositions of reduced biquaternion matrices. IEEE Trans. Circ. Syst. I., 55(9), 1549-8328, (2008).

[5] Isokawa, T., Nishimura, H., Matsui, N.: Commutative quaternion and multistate hopfield neural networks. In Proc. Int. Joint Conf. Neural Netw., Barcelona, Spain, 1281-1286, (2010).

[6] Kosal H. H., Akyigit M., Tosun M.: Consimilarity of commutative quaternion matrices. Miskolc Math. Notes, 16(2), 965-977, (2015).

[7] Yuan, S. F., Tian, Y., Li, M. Z.: On Hermitian solutions of the reduced biquaternion matrix equation $(A X B, C X D)=(E, G)$. Linear Multilinear Algebra, 1-19 (2018). DOI: 10.1080/03081087.2018.1543383.

[8] Kosal H. H., Tosun M.: Universal similarity factorization equalities for commutative quaternions and their matrices. Linear Multilinear Algebra, 67(5), 926-938, (2019).

[9] Kosal H. H.: Least-squares solutions of the reduced biquaternion matrix equation $A X=B$ and their applications in colour image restoration. J. Modern Opt., 66(18), 1802-1810, (2019).

[10] Zhang D., Guo Z., Wang G., Jiang T.: Algebraic techniques for least squares problems in commutative quaternionic theory. Math Meth Appl Sci., $43,3513-3523,(2020)$.

[11] Catoni, F., Cannata, R., Zampetti, P.: An introduction to commutative quaternions. Adv. Appl. Clifford Algebras, 16, 1-28, (2006). 


\section{Affiliations}

HidAYET Huda Kosal

ADDRESS: Sakarya University, Dept. of Mathematics, 54050, Sakarya-TURKEY.

E-MAIL: hhkosal@sakarya.edu.tr

ORCID ID: 0000-0002-4083-462X

TUCE BILGILI

ADDRESs: Sakarya University, Dept. of Mathematics, 54050, Sakarya-TURKEY.

E-MAIL: tuce.bilgili1@ogr.sakarya.edu.tr

ORCID ID: 0000-0002-9554-65027 